\title{
Motion Controller Realizing Cyclic Ball Passing Strategy among Multiple Mobile Robots in Robot Soccer Games
}

\author{
Tzu-Chen Liang and Jing-Sin Liu \\ Institute of Information Science 20 \\ Academia Sinica \\ Nankang, Taipei 115, Taiwan, R.O.C. \\ liu@iis.sinica.edu.tw
}

\begin{abstract}
A motion control and the corresponded strategy to realize cyclic ball passing motion in robot soccer games are presented in this paper. By this strategy, multiple mobile robots kick the ball in turn with high speed while they adjust or change their formation. The ball-kick controller is to drive the robot to an adequate position with a prescribed velocity in a fixed finite time. After kicking, the robot is driven to a suitable position for next ball-passing movement and could accomplish other objectives in robot soccer games. A computer simulation using dynamical model of two-wheeled mobile robot demonstrates the feasibility of this method.
\end{abstract}

\section{Introduction}

In recent years, robot soccer game enforces research issues such as multi-agent systems, multi-robot cooperative teams, autonomous navigation, sensor fusion, fast pattern recognition and vision-based real time control. It also has been proposed as a benchmark problem for the artificial intelligence and robotic systems. The strategic problems, like policymaking or automatic learning, could be considered as a simulation of human brain; the control issues are like the ability of body movement. Then an intermediate level issue, which is similar to skills of human soccer players, exists and needs to be further investigated.

In a robot soccer game, the actions of a robot can be divided into several sub-goals [4][7], such as shoot, pass, dribble or block. Because the situation changes fast, these sub-goals are extremely time dependent. Many controllers for wheeled mobile robots are proved asymptotically or exponentially stable and can be applied to posture or path tracking problem well [6][8][10]. However, for a movement like ball passing in robot soccer games, we need a global strategy for path planning of each robot and the generated path should be judged practicable and can be executed in time [5]. The ball should be passed as fast as possible lest it would be intercepted by the other team. Therefore the kick movement should be simple so that can be completed in short time.

In this paper we suggests a kick control that only drives the robot forward, i.e. the driving torques of two wheels are the same. This control actuates a straight-line motion for the robot, and is useful in the robot soccer game due to its prerogatives: fast and reliable. Once the computer program predicts the trajectory of the ball and finds the kick position and time, this controller can drive the robot to achieve this position on the time with a prescribed velocity. The moving direction of ball after being kicked is limited controlled by the prescribed robot velocity.

Using this kick control, enable us to develop a novel ball-passing strategy. Multiple mobile robots arranged as a geometric formation can pass the ball in turn as a cycle. Each robot devotes part of time to prepare and pass the ball, the idle time is planned to adjust its relative position and orientation, or to move or change the formation.

We will focus on the ball passing and control problem, the velocities and positions of the robots and ball at present time are assumed known by sensor. In the next section, the ball passing strategy is introduced and explained. The ball-kick controller is given in section III. Section IV is a computer simulation using dynamical model of two-wheeled mobile robot to demonstrate the passing strategy and ball-kick controller. Finally, a conclusion will be addressed.

\section{Ball Passing Strategy}

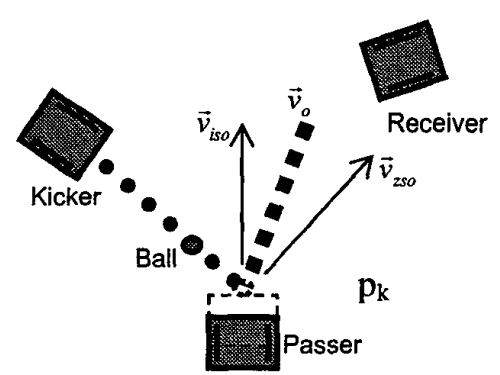

Fig. 1 The relative positions of Kicker, Passer and Receiver. $\vec{v}_{z s o}$ is the velocity direction of ball after a zero-speed collision with passer, and $\vec{v}_{i s o}$ is that after an infinite-speed collision. $\vec{v}_{o}$ is the actual velocity direction of the ball after kicked by passer.

The ball passing strategy presented here is a combined trajectory planning/control method that real time generates the trajectory of multiple mobile robots to pass the ball among them in turn. In a passing cycle, each robot does not hold the ball. Instead, it kicks the ball by the front surface, which is orthogonal to the moving 
direction of robot. For a ball passing movement, three robots are considered, named passer, kicker and receiver as shown in figure 1. The passer is the robot that intends to kick the moving ball coming from the kicker direction to the receiver direction. When the ball collides with the passer, the roles change: The passer becomes the kicker, the receiver becomes the passer, and the new receiver is prescribed by strategy, as shown in figure 2 .

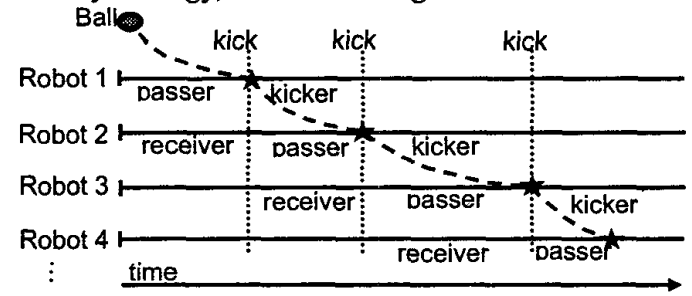

Fig. 2 The conceptual diagram of ball passing strategy. Dashed line is the ball trajectory and each star sign means that a robot kicks the ball. After kicking, roles change as shown.

Three parts of the ball passing strategy are described here.

a. Passer: Predict and kick

The velocity vector of the ball is observed by sensor after it kicked by the kicker, and a motion predictor predicts its trajectory. The ball motion is assumed known by physical model or looking table. The robot trajectory to kick the moving ball is designed as a straight line due to the controller restriction. Therefore the kick position can be computed by geometry. Ball motion predictor generates the kick time $\left(t_{f}\right)$ and position $\left(s_{f}\right)$. The kick velocity $\left(\dot{s}_{f}\right)$ of the passer robot can be determined arbitrary according to the expectative trend of ball passing speed. Therefore, the ball-kick problem becomes a path planning and control problem. We will discuss this in section III.

b. Receiver: Prepare for passing

Because the collision reaction computation is not included in the ball motion predictor, the preparation for a passing can be only started after the ball kicked by the kicker. Actually, the moving direction of ball after collision is not easy to predict before it happened in real game owing to lack of exact collision model and other uncertainties. However, in fast passing situation, a limited prediction is required.

We have known by dynamics that when the kick speed is zero, the ball reflects with an angle equal to the angle of incidence. Moreover, if the kick speed is infinite, the ball is kicked to the direction of robot velocity. Thus, the velocity direction $\left(\vec{n}_{0}\right)$ of the ball after a kick must lie between the zero-speed kicked direction $\left(\vec{n}_{2 s o}\right)$ and the infinite-speed kicked direction $\left(\vec{n}_{i s o}\right)$, as shown in figure 1 . This can be expressed as

$$
\begin{aligned}
& \vec{n}_{o}=m \vec{n}_{z s o}+(1-m) \vec{n}_{i s o} \\
& \text { with } 0 \leq m \leq 1
\end{aligned}
$$

By (1), after each kick, a weighting parameter $m$ can be computed by the measurable $\vec{n}_{o}, \vec{n}_{z s o}$ and $\vec{n}_{i s o}$. The physical meaning of $m$ is the ratio of contribution of robot kick speed to the reflection direction change. In general we hope the ball speed keeps stable, and the robot kick speed which is decided according to ball speed is also not change a lot. Then the $m$ computed in the previous kick of this robot could be a reference value to make a prediction of $\vec{n}_{o}$ when the ball has not been kicked by passer in this passing cycle. Combining with the direction of the receiver, the receiver's kick position is a geometric computation of intersectional point of two lines. Different from passer, this prediction, without kick time information, is only a reference for preparation. When a robot acts as a receiver, the controller drives it to the standby position, which is direct behind the predicted kick position by a suitable distance, until it becomes a passer.

\section{c. Kicker and others: Do Other Things}

If a robot does not act the role as a passer or receiver, it could change its position and orientation according to global strategy to accomplish some other goals, such as moving or formation change. It is a dilemma that if the movement of each robot with respect to the formation is larger, then the updated $m$ is less accurate in the standby position prediction of receiver, and the passing speed is also constrained.

The initial position and orientation of robots are important due to the strategy use only straight motion. Robots should approximately form a passing formation. Once the passing movement starts, robots pass the ball in turn, and the formation changes gradually by global strategy. Generally speaking, the formation change affects the time duration of each ball passing movement. This effect should be considered to keep the passing movement stable. Although the kick velocity can be arbitrary assigned under the restriction of ball-kick control, it would be better decided according to ball speed to avoid the relentless increasing or deceasing ball speed

\section{Ball-Kick Control}

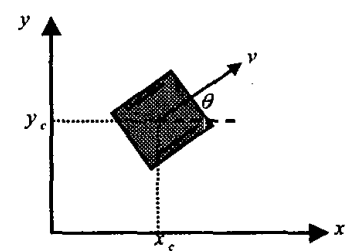

Fig. 3 The schematic representation of the mobile robot. The unicycle mobile robot is shown in figure 3 , this 
kind of robot is mostly used in robot soccer games, but the kick control suggested here is not restricted in this kind of robot. The vehicle position is described by the coordinate $(x, y)$ of the mid point between the two driving wheels, and by the orientation angle $\theta$ with respect to a fixed frame. Under the hypothesis of "pure rolling" and "non slipping", the vehicle satisfies the nonholonomic constraint,

$$
\dot{x} \sin (\theta)-\dot{y} \cos (\theta)=0
$$

The dynamical model of vehicle is described by the following equations [8],

$$
\begin{gathered}
\ddot{x}=-\sin (\theta)[\dot{x} \cos (\theta)+\dot{y} \sin (\theta)] \dot{\theta}+\frac{\cos (\theta)}{m r}\left(\tau_{l}+\tau_{r}\right) \\
\ddot{y}=\cos (\theta)[\dot{x} \cos (\theta)+\dot{y} \sin (\theta)] \dot{\theta}+\frac{\sin (\theta)}{m r}\left(\tau_{l}+\tau_{r}\right) \\
\ddot{\theta}=\frac{R}{l r}\left(\tau_{l}-\tau_{r}\right)
\end{gathered}
$$

Where $\tau_{l}$ and $\tau_{r}$ are driving torques of left and right wheels; $m, I, R$ are the robot mass, moment of inertia and length respectively, $r$ is the wheel radius.

The system of equations (3) is nonlinear, and to design a controller is not easy. Hence we restrict the kick trajectory to a straight line. Let $\tau_{i}=\tau_{r}=u / 2$ and $s$ be the traveling distance, then ( 3 ) is reduced to a second-order linear system,

$$
\ddot{s}=\frac{u}{m r}
$$

The ball-kick control problem is in fact a finite-time control problem: to find an input $u(s, \dot{s}, t)$, such that at time $t_{f}$, the robot should be at the position $s_{f}$ with the velocity $\dot{s}_{f}$. This can be converted to a classical tracking control problem if the controller restricts the tracking error at time $t_{f}$ to be small by setting the rate of convergence properly.

A tracking problem is that for a given reference path $s_{r}(t)$, to find a control input $u(s(t), \dot{s}(t), t)$, in order that

$$
\lim _{r \rightarrow \infty}\left(s(t)-s_{r}(t)\right)=0
$$

In our case, it is required that for bounded initial errors $\left|s(0)-s_{r}(0)\right|$ and $\left|s(0)-\dot{s}_{r}(0)\right|$, at time $t_{f}$,

$$
\left|s\left(t_{f}\right)-s_{r}\left(t_{f}\right)\right|<\delta_{1} \text { and }\left|\dot{s}\left(t_{f}\right)-\dot{s}_{r}\left(t_{f}\right)\right|<\delta_{2}
$$

where $\delta_{1}$ and $\delta_{2}$ are small enough to not cause ball losing. By definition, if a system is exponential stable, there exist two strictly positive numbers, $\alpha$ and $\lambda$ such that

$$
\forall t>0,\|\tilde{e}(t)\| \leq \alpha\|\tilde{e}(0)\| \mathrm{e}^{-\lambda t}
$$

where $\tilde{e}(t)$ is the error.

$$
\tilde{e}(t)=\left[\begin{array}{l}
e(t) \\
\dot{e}(t)
\end{array}\right]=\left[\begin{array}{l}
s(t)-s_{r}(t) \\
s(t)-\dot{s}_{r}(t)
\end{array}\right]
$$

as tracking errors. If we design a controller which makes the system exponentially stable, then $\alpha$ and $\lambda$ in (7) can be chosen to assure the requirement of $(8)$, that is

$$
\alpha\|\tilde{e}(0)\| \mathrm{e}^{-\lambda t_{s}}<\max \left(\delta_{1}, \delta_{2}\right)
$$

Choose the exponential stable error dynamics as

$$
\ddot{e}(t)+k_{2} \dot{e}(t)+k_{1} e(t)=0
$$

where $k_{1}$ and $k_{2}$ are positive numbers.

$$
\begin{aligned}
\frac{u}{m r} & =\ddot{s}(t) \\
& =\ddot{s}_{r}(t)+\ddot{e}(t) \\
& =\ddot{s}_{r}(t)-k_{2} \dot{e}(t)-k_{1} e(t) \\
& =\ddot{s}_{r}(t)-k_{2}\left(\dot{s}(t)-\dot{s}_{r}(t)\right)-k_{1}\left(s(t)-s_{r}(t)\right)
\end{aligned}
$$

Assume $s_{r}(t)$ is known, $\mathbb{C}^{2}$ continuous, use (11) as the controller, the exponential tracking is achieved. Furthermore, if we set the reference path properly to let the initial error be zero, the condition of equation (9) is satisfied automatically and $k_{1}$ and $k_{2}$ can be chosen arbitrary. This kind of reference path $s_{r}(t)$ is defined by four boundary conditions,

$$
\begin{aligned}
& s_{r}(0)=s_{0} \equiv 0, s_{r}\left(t_{f}\right)=s_{f}, \\
& \dot{s}_{r}(0)=\dot{s}_{0}, \text { and } \dot{s}_{r}\left(t_{f}\right)=\dot{s}_{f}
\end{aligned}
$$

$s_{0}$ and $\dot{s}_{0}$ are the current position and velocity of the robot. We set $s_{r}(t)$ to be a 3-order polynomial,

$$
s_{r}(t)=q_{1} t^{3}+q_{2} t^{2}+q_{3} t+q_{4}
$$

the four coefficients can be solved as

$$
\begin{aligned}
& q_{1}=\frac{\dot{s}_{f}{ }^{\prime} t_{f}-2 s_{f}^{\prime}}{t_{f}^{3}}, q_{2}=\frac{3 s_{f}{ }^{\prime}-\dot{s}_{f}{ }^{\prime} t_{f}}{t_{f}^{2}}, \\
& q_{3}=\dot{s}_{0}, \text { and } q_{4}=0
\end{aligned}
$$

where $s_{f}^{\prime}=s_{f}-\dot{s}_{0} t_{f}$ and $\dot{s}_{f}{ }^{\prime}=\dot{s}_{f}-\dot{s}_{0}$.

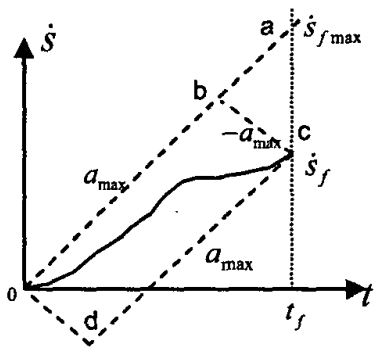

Fig. 4 The velocity-time diagram of a kick movement.

Note that the control input $u(s(t), \dot{s}(t), t)$ is not unlimited in general so that the kick movement has its theoretical bound. When the planned velocity profile has slope exceeding the maximal acceleration of robot, the track may be failure. Figure 4 is velocity-time graph of a kick movement. Line 0 -a means the robot dashing in its maximal acceleration $\left(a_{\max }\right)$. The integrated area of line 0 -a with respect to time is the maximal distance that the robot can move in finite time $t_{f}$. For a fixed $t_{f}$, an $\dot{s}_{f}$ (point c) has its theoretical maximal and minimal 
driving distance corresponded to line $0-b-c$ and $0-d-c$. This two area is computed as,

$$
\begin{aligned}
& s_{f \max }\left(\dot{s}_{f}\right)=\frac{a_{\max }}{4}\left(\frac{\dot{s}_{f}}{a_{\max }}+t_{f}\right)^{2}-\frac{\dot{s}_{f}^{2}}{2 a_{\max }} \\
& s_{f \min }\left(\dot{s}_{f}\right)=\frac{\dot{s}_{f}^{2}}{2 a_{\max }}-\frac{a_{\max }}{8}\left(t_{f}-\frac{\dot{s}_{f}}{a_{\max }}\right)^{2}
\end{aligned}
$$

For testing the effect of the suggested control law, a simulation is performed. The maximal acceleration, which is a function of wheel driving torque and robot mass, is set to be $3 \mathrm{~m} / \mathrm{sec}^{2} . k_{1}=15$ and $k_{2}=15$. $t_{f}$ is $0.5 \mathrm{sec}$ because it is the desired average passing duration. We simulate all the probable combination of $s_{f}$ and $\dot{s}_{f}$ with a grid interval of $5\left(\mathrm{~m}\right.$ in $s_{f}$, and $\mathrm{m} / \mathrm{sec}$ in $\left.\dot{s}_{f}\right)$, and define the attainable case as,

$a b s\left(s\left(t_{f}\right)-s_{f}\right)<0.001 \mathrm{~m}$ and $a b s\left(\dot{s}_{f}\left(t_{f}\right)-\dot{s}_{f}\right)<0.001 \mathrm{~m} / \mathrm{sec}$

Which means the position error should be less then $1 \mathrm{~mm}$, and the velocity error should be less then $1 \mathrm{~mm} / \mathrm{sec}$. Figure 5 shows the attainable region of the kick controller. $x$-axis and $y$-axis represented $\dot{s}_{f}$ and $s_{f}$ respectively, and two dotted line computed by equation (15) are the theoretical limitation of a kick movement. This robot in time $t_{f}$ cannot achieve area outside the dotted lines.

From the result of figure 5 , for a predicted kick position in front of the robot by a distance $s_{f}$, an attainable range of $\dot{s}_{f}$ can be selected appropriately.

The above controller is designed under the assumption that the robot always move along the planned trajectory. Though we plan each trajectory as a straight line and the driving torques of two wheels remain the same, it is not promised that the deviation would not happen. Therefore another two errors are defined: $e_{d}$ is the signed distance between the planned trajectory and the robot; $e_{\theta}$ is the angle between the tangent direction of the planned trajectory and the moving direction of the robot. Then a difference torque is defined as

$$
\tau_{\Delta}(t)=k_{3} e_{d}(t)+k_{4} e_{\theta}(t)
$$

The input torques of left and right wheels are

$$
\begin{aligned}
& \tau_{R}(t)=\frac{1}{2}\left(\frac{u(t)}{m r}-\tau_{\Delta}(t)\right) \\
& \tau_{L}(t)=\frac{1}{2}\left(\frac{u(t)}{m r}+\tau_{\Delta}(t)\right)
\end{aligned}
$$

\section{Simulation}

The robot simulator is an imitation of Simurosot software of FIRA [1], except that the robot motion is simulated by dynamical model as in equation (3), not the kinematical one in Simurosot.

The kick controller is applied to drive the robot to kick the ball when it is assigned as a passer and to drive the robot back to the original position when it is assigned as a kicker. The shift motion, as shown in figure 6 and 7 . We set the center in $70 \mathrm{~mm}$ front of each robot. By updating the position of the center and the orientation of the robot, the formation can be controlled. Note the motion in idle time is not necessary to be a concentric shift motion, but it is helpful for a global formation change or moving, as we'll show below.

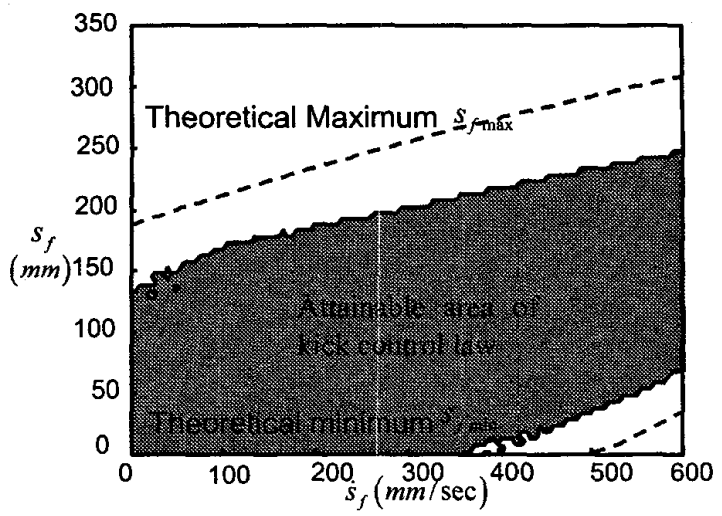

Fig. 5 The attainable area of the suggested kick control law.

Figure 6 is a close look of a kick movement. The ball comes from the left side, and the ball-kick controller drives the robot to kick it. After kicking, the controller activates to drive back the robot with a shift.

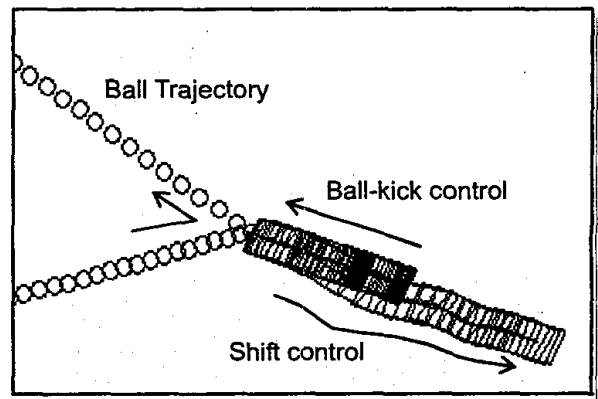

Fig. 6 A close look of ball-kick action.

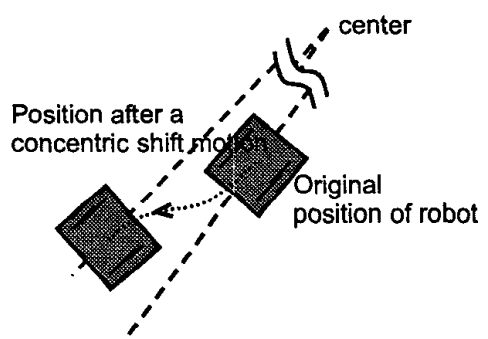

Fig. 7 The concentric shift motion of robot.

\section{Simulation 1}

In this simulation, three identical mobile robots pass the ball in turn. Between each passing movement, robots try to adjust the formation to a right triangle. This includes correcting their relative angle to $2 \pi / 3$, and updating their shift motion centers to appropriate positions. Initial position and orientation $(x, y, \theta)(x, y$ in 
$m m$ and $\theta$ in radian) of three robots are $A:(200,1100,-1.88)$, B: $(1400,700,-2.82)$ and $C:(600,190,1.42)$. Initial position and velocity of ball are $(600,500)$ and $(-375,708)$ as shown in figure 8 . The robot length and maximal acceleration are $80 \mathrm{~mm}$ and $5 \mathrm{~m} / \mathrm{sec}^{2}$ respectively. Ball radius is $10 \mathrm{~mm}$.

The preparation position of receiver is behind the predicted kick position by $200 \mathrm{~mm}$, and the kick velocity $\dot{s}_{f}$ is set from 200 to $580 \mathrm{~mm} / \mathrm{sec}$ to avoid exceeding the attainable region of controller. Inside this range, $\dot{s}_{f}$ is chosen inversely proportional to the ball speed, i.e., the higher the ball speed, the lower the kick speed, to keep the passing movement steady.

Figure 9 and 10 shows the simulated traces of each robot and ball in the first 15 and 50 to 60 passing movements. In figure 9 , the movement of each passing action is large, and the locus of ball is not repetitive. After 50 passing movements, as shown in figure 10 , they form a right triangle and the locus of ball is almost the same in every cycle. Besides, the average time duration of each passing decreases to $0.5 \mathrm{sec}$.

Simulation 2

Based on simulation one, an extra duty is assigned to each robot at their idle time. Which is to keep the formation and rotate with respect to the co-center. The rotation angle during each passing cycle is 0.02 in radian. The rotation starts after the $60^{\text {th }}$ passing movement. Figure 11 shows the traces of robots and ball from 100 to 120 passing action; figure 12 is the traces from 200 to 220 passing action. Note the formation has rotated an angle about 0.67 without changing the relative position of each robot.

To clearly observe the traces of three robots, figure 13 shows only the traces of their locations in the first 300 passing movements. The points in the center of this figure are the traces of center of their shift motion.

In the two simulations, the initial $m$ values of three robots are set to be 0.9 , and updated after every passing movement by equation (2). Figure 14-a and $b$ show the change of $m$ with respect to passing cycle in these two simulations. Note that three passing movements are one passing cycle. In figure 14-a, the $m$ values of three robots drop from 0.9 to about 0.5 in the first 150 passing cycles, and change little after it. This means use equation (1) and (2) to rough predict the ball's moving direction after collision in ordinary passing cycles are effectual. In figure $14-b$, the rotation starts at the $60^{\text {th }}$ passing movement, i.e. the $20^{\text {th }}$ passing cycle. The $m$ values also drop, and oscillate in a range of about 0.1 . This means the prediction is also useful in a regular formation rotation like simulation 2 .

If we set $m$ as constant 0.9 in each simulation, robots have much redundant motion and are easy to miss ball. But if apply the steady average values $m(0.5$ and
0.6 respectively), the passing movements are also fluent as the two simulations.

\section{Conclusion}

In this paper, we present a practical ball-passing strategy for multiple robots in soccer games. The passing movement, which keeps the ball in a high speed without holding, is useful in real robot soccer games. The passing strategy uses only the straight motion of robot to kick ball and during the idle time, a robot can execute other jobs. To realize the strategy, the corresponded control law is also suggested.

Conventional researches of mobile robot control focus on posture or path tracking problem of mobile robots, but most movements in robot soccer games are more adequate to formulate as a final state control problem. This paper expresses this issue by a ball-passing problem. The suggested control law can be also applied to other movements in robot soccer game like shoot or block.

The future work is to combine more complex intention in the passing movement, like the receiver selection or large-range formation change. Besides, the ball-passing controller needs to extend to let the orientation controllable and its attainable region should be enlarged.

\section{References}

[1] Hong Bingrong, Gao Quansheng, etc., "Robot Soccer Simulation Competition Platform Base on Multi-agent", FIRA-KAIST Cup Workshop, May, 2000

[2] Yutaka Kanayama and Shin'ichi Yuta, "Vehicle Path Specification by a Sequence of Straight Lines", IEEE Journal of Robotics and Automation, vol. 4, no. 3, June, 1988, pp. 265-276.

[3] Byoung-Ju Lee and Gwi-Tae Park, "A Robot in Intelligent Environment: Soccer Robot", Procceedings of the 1999 IEEE/ASME International Conference on Advanced Intelligent Mechatronics, Sep., 1999.

[4] Barry Brian Werger, "Principles of Minimal Control for Comprehensive Team Behavior", Proceedings of the 1998 IEEE International on Robotics \& Automation, May, 1998.

[5] Hiroaki Yamaguchi and Joel W. Burdick, "Time-Varying Feedback Control for Nonholonomic Mobile Robots Forming Group Formations", Proceedings of the $37^{\text {th }}$ IEEE Conference on Decision \& Control, Dec., 1998.

[6] Byoung-Ju Lee, Sung-Oh Lee and Gwi-Tae Park, "Trajectory Generation and Motion Tracking Control for the Robot Soccer Game", Proceedings of the 1999 IEEE/RSJ International Conference on Intelligent Robots and Systems, 1999. 


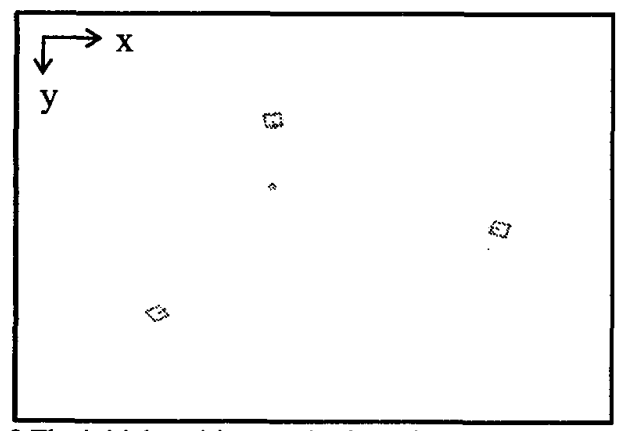

Fig. 8 The initial positions and orientations of robots and ball.

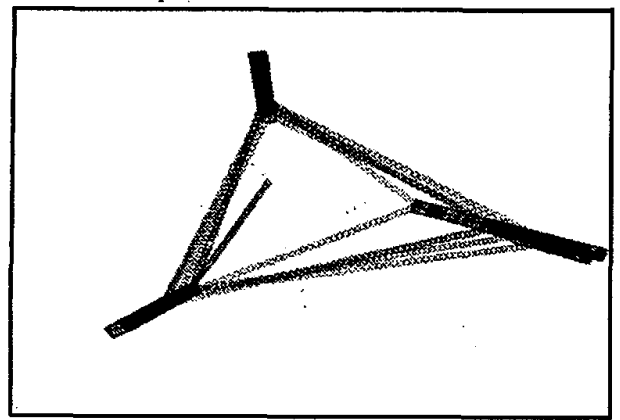

Fig. 9 The traces of robots and ball in the first 15 passing movements of simulation 1 .

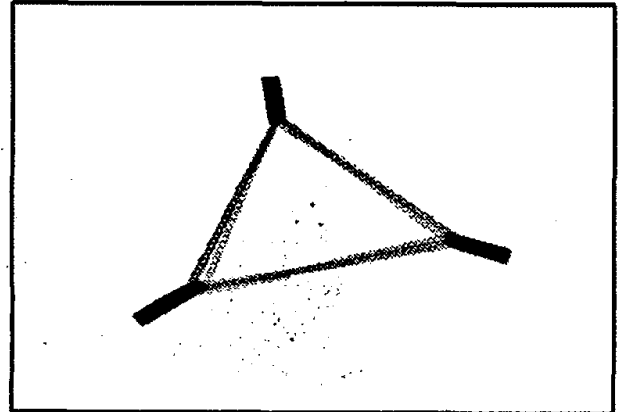

Fig. 10 The traces of robots and ball in the 50 to 60 passing movements of simulation 1.

[7] Xuming Xu, Shi Li, etc., "A Survey: RoboCup and the Research", Proceedings of the $3^{\text {rd }}$ World Congress on Intelligent Control and Automation, June, 2000.

[8] M. L. Corradini and G. Orlando, "Robust Tracking Control of Mobile Robots in the Presence of Uncertainties in the Dynamical Model", Journal of Robotic Systems, vol. 18, no. 6, June, 2001, pp. 317-323.

[9] Jong-Hwan Kim, Kwang-Choon Kim, etc. "Path Planning and Role Selection Mechanism for Soccer Robots", Proceedings of the 1998 IEEE International on Robotics \& Automation, May, 1998.

[10] Jun-ichi Imura, Keigo Kobayashi, and Tsuneo Yoshikawa, "Exponential Stabilization Problem of Nonholonomic Chained Systems with Specified Transient Response", Proceedings of the $35^{\text {th }}$ Conference on Decision and Control, Dec., 1996.

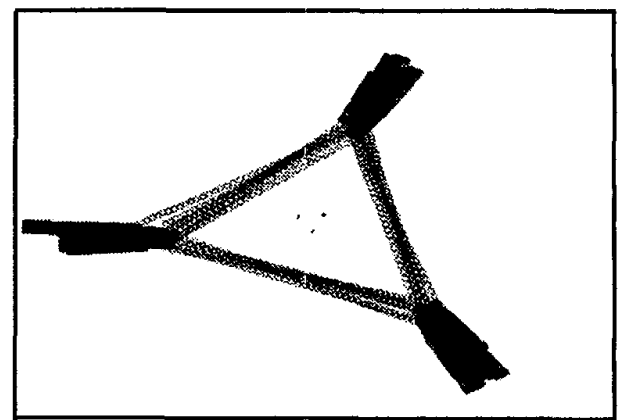

Fig.11 The traces of robots and ball in the 100 to 120 passing movements of simulation 2 .

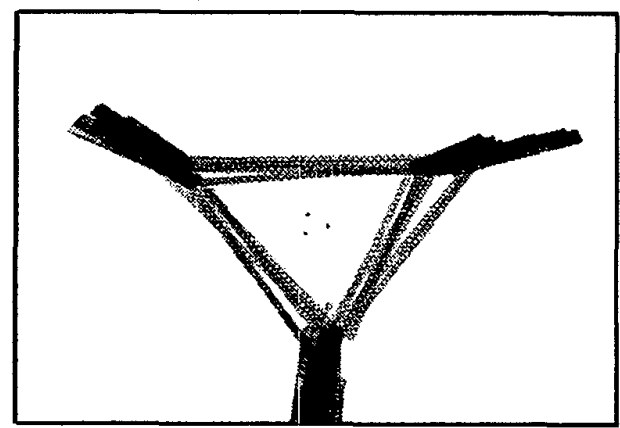

Fig. 12 The traces of robots and ball in the 200 to 220 passing movements of simulation 2 .

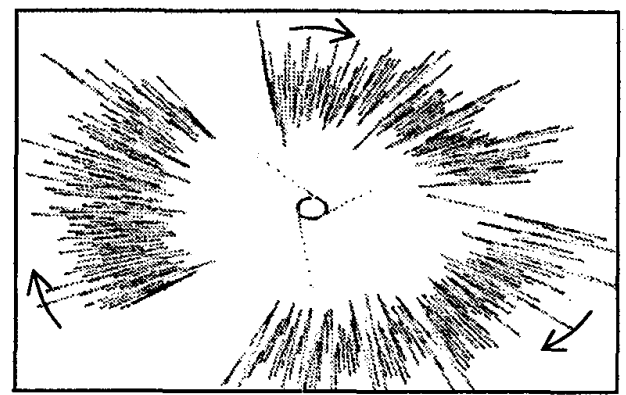

Fig. 13 Traces of locations and center of shift motion of robots in the first 300 passing movements.

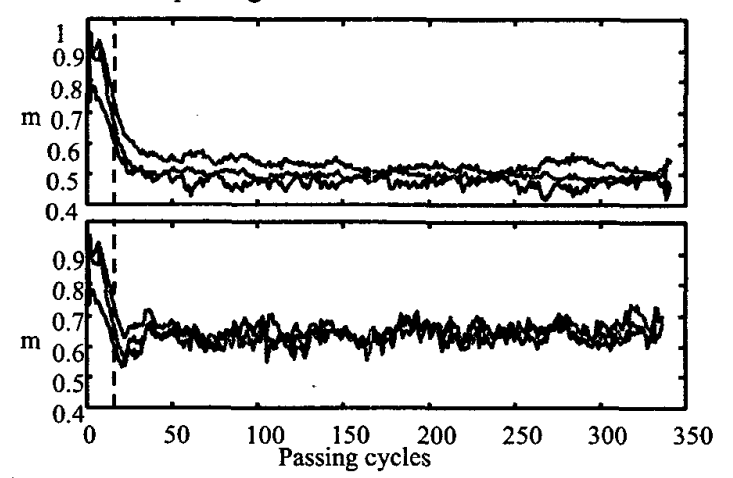

Fig. 14 The $m$ value-passing cycles plots. Figure $14-a$ is simulation 1, and figure 14-b is simulation 2. Dashed line marks the $20^{\text {th }}$ passing cycle. The curves of $m$ between 0 to 20 cycles are the same in two simulations. 